\title{
Calculating the running coupling in strong electroweak models
}

\section{Zoltán Fodor}

Department of Physics, University of Wuppertal, Gauss Strasse 20, D-42119, Germany

and

Institute for Theoretical Physics, Eotvos University Budapest, Pazmany P. 1, H-1117, Hungary

E-mail: fodor@bodri.elte.hu

\section{Kieran Holland*}

Department of Physics, University of the Pacific

3601 Pacific Ave, Stockton CA 95211, USA

E-mail: khollandepacific.edu

\section{Julius Kuti}

Department of Physics 0319, University of California, San Diego

9500 Gilman Drive, La Jolla CA 92093, USA

E-mail: jkuti@ucsd.edu

\section{Dániel Nógrádi}

Department of Physics 0319, University of California, San Diego 9500 Gilman Drive, La Jolla CA 92093, USA

E-mail: nogradi@bodri.elte.hu

\section{Chris Schroeder}

Department of Physics 0319, University of California, San Diego

9500 Gilman Drive, La Jolla CA 92093, USA

E-mail: crs@physics.ucsd.edu

One possibility for Beyond Standard Model physics is a new strongly-interacting gauge theory. One way to determine if a non-abelian gauge theory is QCD-like or conformal is to measure the running of the renormalized gauge coupling. We define the renormalized coupling from Wilson loop ratios, and measure these ratios via lattice simulations. We test this method in $S U(3)$ pure gauge theory and show some first results for simulations with dynamical fermions in the fundamental representation.

The XXVII International Symposium on Lattice Field Theory

July 26-31, 2009

Peking University, Beijing, China

\footnotetext{
${ }^{*}$ Speaker.
} 


\section{Running coupling}

There is great interest in the possibility that Beyond Standard Model physics might take the form of new strongly coupled gauge theories [1]-[21], one example being technicolor. For model building, it is necessary to distinguish conformal theories from those with a mass gap like QCD. There have been a number of lattice studies of this issue [23]-[32]. One signal is the running coupling, which has an infrared fixed point in a conformal theory. We developed our method for the running coupling and its beta function from the definition of the continuum renormalized coupling constant using the second derivative of $R \times T$ Wilson loops [24]. This coupling runs with the size of the Wilson loop in infinite volume [33]. We generalized this definition to a finite volume $L^{4}$ keeping $R / L$ fixed and run the coupling with $L$ as in the Schrodinger functional method. A similar method was developed independently in [32].

Consider Wilson loops $W(R, T, L)$, where $R$ and $T$ are the space-like and time-like extents of the loop, and the lattice volume is $L^{4}$ (all dimensionful quantities are expressed in units of the lattice spacing $a$ ). A renormalized coupling can be defined by

$$
g^{2}(R / L, L)=-\left.\frac{R^{2}}{k(R / L)} \frac{\partial^{2}}{\partial R \partial T} \ln \langle W(R, T, L)\rangle\right|_{T=R},
$$

where for convenience the definition will be restricted to Wilson loops with $T=R$, and $\langle\ldots\rangle$ is the expectation value of some quantity over the full path integral. This definition can be motivated by both renormalized and bare perturbation theory, where the leading term is the tree-level coupling. The renormalization scheme is defined by holding $R / L$ to some fixed value. The quantity $k(R / L)$ can be calculated from Wilson loop expectation values using perturbation theory. This is done numerically on finite lattices, hence $k$ contains lattice artifacts which vanish as $L \rightarrow \infty$. The role of lattice simulations is to measure the expectation values non-perturbatively. On the lattice, derivatives are replaced by finite differences, so the renormalized coupling is defined to be

$$
\begin{aligned}
& g^{2}((R+1 / 2) / L, L)=\frac{1}{k(R / L)}(R+1 / 2)^{2} \chi(R+1 / 2, L), \\
& \chi(R+1 / 2, L)=-\left.\ln \left[\frac{W(R+1, T+1, L) W(R, T, L)}{W(R+1, T, L) W(R, T+1, L)}\right]\right|_{T=R},
\end{aligned}
$$

where $\chi$ is the Creutz ratio [34], and the renormalization scheme is defined by holding the value of $r=(R+1 / 2) / L$ fixed.

With this definition, the renormalized coupling $g^{2}$ is a function of the lattice size $L$ and the fixed value of $r$. The continuum limit corresponds to $L \rightarrow \infty$, where the physical length scale $L_{\text {phys }}$ is held fixed while the lattice spacing $a \rightarrow 0$. The coupling is non-perturbatively defined, as the expectation values are calculated via lattice simulations, which integrate over the full phase space of the theory. One starts the RG flow from some reference physical point $L_{\mathrm{phys}, 0}$ which is set by the choice e.g. $g^{2}\left(r, L_{\text {phys }, 0}\right)=0.8$. In a QCD-like theory, $g^{2}$ increases with increasing $L_{\text {phys }}$ flowing in the infrared direction. In a conformal theory, $g^{2}$ flows towards some non-trivial infrared fixed point $g^{* 2}$ as $L_{\text {phys }}$ increases, whereas in a trivial theory, $g^{2}$ decreases with $L_{\text {phys }}$.

One way to measure the running of the renormalized coupling in the continuum limit is via step-scaling. The bare lattice coupling is defined in the usual way $\beta=6 / g_{0}^{2}$ as it appears in the 

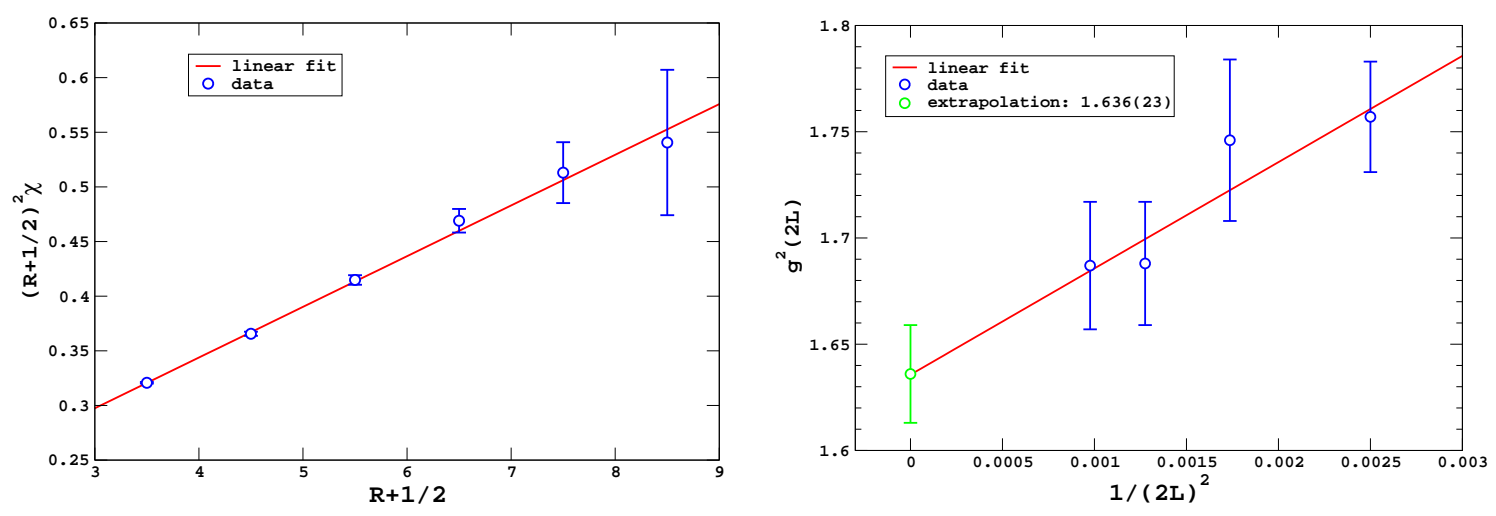

Figure 1: (Left) The rescaled Creutz ratio on a $28^{4}$ lattice at $\beta=6.99$. We interpolate the data linearly to $r=(R+1 / 2) / L=0.25$, giving a chi squared per degree of freedom 2.0/4. (Right) The measured coupling $g^{2}\left(2 L_{i}, \beta_{i}\right)$ for $2 L_{i}=20,24,28$ and 32 , where $\beta_{i}$ is tuned such that $g^{2}\left(L_{i}, \beta_{i}\right)=1.44$. A linear continuum extrapolation gives $g^{2}\left(2 L_{\text {phys }}\right)=1.636(23)$, with $\chi^{2} /$ dof $=0.57 / 2$.

lattice action. On a sequence of lattice sizes $L_{1}, L_{2}, \ldots, L_{n}$, the bare coupling is tuned on each lattice so that exactly the same value $g^{2}\left(r, L_{i}, \beta_{i}\right)=g^{2}\left(r, L_{\text {phys }}\right)$ is measured via simulations. Next a new set of simulations is performed, on a sequence of lattice sizes $2 L_{1}, 2 L_{2}, \ldots, 2 L_{n}$, using the corresponding tuned couplings $\beta_{1}, \beta_{2}, \ldots, \beta_{n}$. From the simulations, one measures $g^{2}\left(r, 2 L_{i}, \beta_{i}\right)$, which vary with the bare coupling i.e. the lattice spacing. These data are extrapolated to the continuum as a function of $1 / L_{i}^{2}$. This gives one blocking step $g^{2}\left(r, L_{\text {phys }}\right) \rightarrow g^{2}\left(r, 2 L_{\text {phys }}\right)$ in the continuum RG flow. The whole procedure is then iterated. The chain of measurements gives the flow $g^{2}\left(r, L_{\text {phys }}\right) \rightarrow g^{2}\left(r, 2 L_{\text {phys }}\right) \rightarrow g^{2}\left(r, 4 L_{\text {phys }}\right) \rightarrow g^{2}\left(r, 8 L_{\text {phys }}\right) \rightarrow \ldots$, as far as is feasible. One is free to choose a different blocking factor, say $L_{\text {phys }} \rightarrow(3 / 2) L_{\text {phys }}$, in which case more blocking steps are required to cover the same energy range.

\section{2. $S U(3)$ pure gauge theory}

As our first test of this method, we study $S U(3)$ pure gauge theory in four dimensions. We simulate using the standard Wilson lattice gauge action, with a mixture of five over-relaxation updates for every heatbath update. We define the renormalization scheme with the fixed value $r=0.25$, for brevity we omit the label $r$ in the renormalized coupling. In this $R / L$ range, the Creutz ratio can be accurately measured, and the geometric factor $k$ converges quickly to its continuumlimit value. In the pure gauge theory test, we actually use the finite $L$ values of $k$, as this may remove some of the cutoff dependence of the renormalized coupling. For the RG flow we choose the blocking step $L \rightarrow 2 L$. We simulate on small lattices of size $L=10,12,14,16,18,20$ and 22 , and the corresponding doubled lattices $2 L=20,24,28,32,36,40$ and 44 . Wilson loop measurements are separated by 1000 sweeps, which we find is sufficient to generate statistically independent configurations.

To tune $\beta_{i}$ for each small lattice size $L_{i}$, we typically run separate simulations at 5 - 10 different $\beta$ values in the relevant range of renormalized coupling $g^{2}$. Each of these runs contains $300-500$ measurements i.e. up to $5 \times 10^{5}$ sweeps. Simulating on the doubled lattices at the tuned $\beta_{i}$ values, 

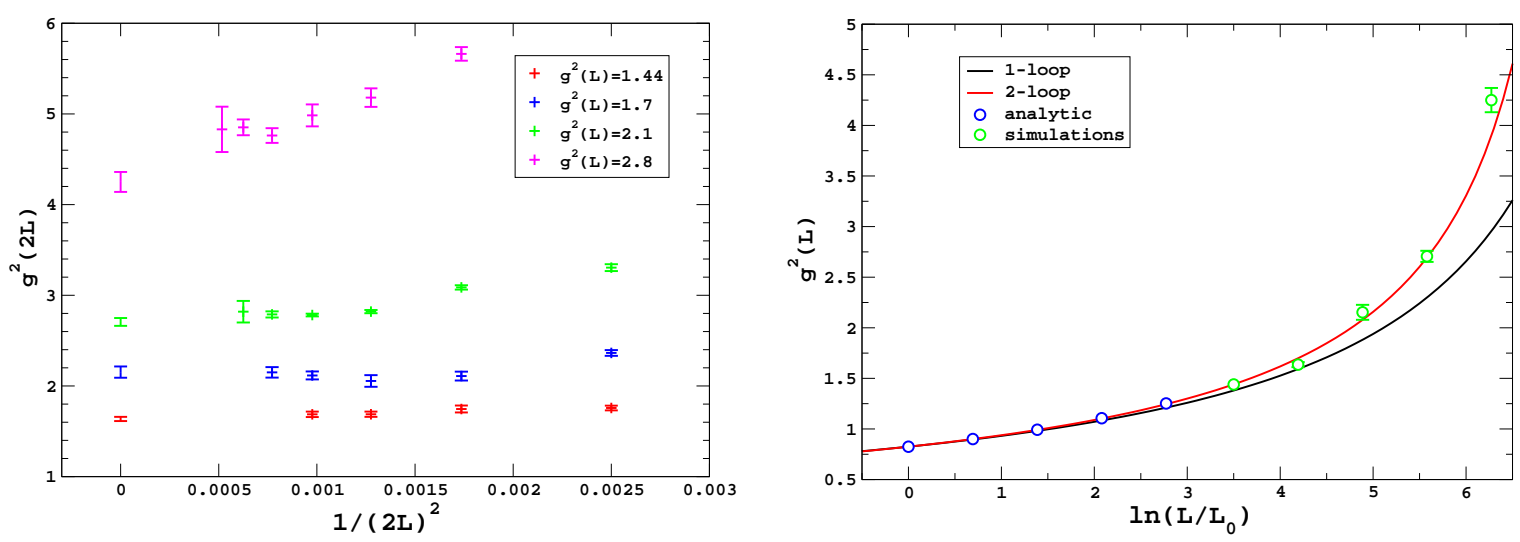

Figure 2: (Left) The continuum extrapolations of four discrete RG steps. (Right) The RG flow $g^{2}\left(L_{\text {phys }}\right)$, combining analytic lattice perturbation theory and the simulation results. The running starts at $g^{2}\left(L_{\mathrm{phys}, 0}\right)=$ 0.825 . There is excellent agreement with continuum 2-loop running, at the strongest coupling, the simulation results begin to break away from perturbation theory.

we generate between 200 and 1000 measurements each, typically more than 500 . The signal of the Creutz ratio disappears into the noise as the size of the Wilson loop increases. One way to suppress the noise in measurements is to gauge fix the configurations to Coulomb gauge, and replace the thin-link Wilson loop with the correlator of the products of the time-like gauge links $[35,36]$. Note that gauge fixing is not implemented in the actual Monte Carlo updating algorithm. An alternative method to suppress noise is to smear the gauge links and measure the fat-link Wilson loop operator. In the pure gauge theory test, we use the gauge-fixing method, in the dynamical fermion simulations we describe later, we use the smearing method. These improvement methods do not correspond to calculating the original thin-link Wilson loop operator.

We show in Fig. 1 (left) a typical result for the rescaled Creutz ratio $(R+1 / 2)^{2} \chi$. The doubled lattice is $28^{4}$ and the bare coupling $\beta=6.99$ is tuned from simulations on $14^{4}$ volumes. Errorbars are calculated using the jackknife method. The renormalized coupling is defined at the point $r=$ $(R+1 / 2) / L=0.25$, corresponding to $(R+1 / 2)=7$ at this lattice size. We interpolate the data linearly to this point, obtaining $\chi^{2} / \mathrm{dof}=2.0 / 4$. The data at different $R$ are highly correlated, being measured on the same gauge configurations. To calculate an error, we bin the gauge configurations, and analyze and interpolate separately each bin, giving a distribution of interpolated values. An example of the step-scaling method is shown in Fig. 1 (right). The bare couplings are tuned such that $g^{2}\left(L_{i}, \beta_{i}\right)=1.44$ for $L_{i}=10,12,14$ and 16 , the figure shows the data $g^{2}\left(2 L_{i}, \beta_{i}\right)$ for $2 L_{i}=$ $20,24,28$ and 32. The leading lattice artifacts in the Creutz ratio are expected to be of order $\mathscr{O}\left(a^{2}\right)$. This corresponds to $\mathscr{O}\left(1 / L^{2}\right)$, since the physical lattice size is $L a$. Extrapolating linearly in $1 /(2 L)^{2}$ gives $g^{2}\left(2 L_{\text {phys }}\right)=1.636(23)$ and $\chi^{2} /$ dof $=0.57 / 2$. For a systematic error, we omit one data point at a time and repeat the extrapolation. Combined in quadrature with the statistical error, our continuum result is $g^{2}\left(2 L_{\text {phys }}\right)=1.636(25)$.

We iterate the procedure, giving four discrete RG steps as shown in Fig. 2 (left). At stronger coupling, we need lattices up to $2 L_{i}=44$ for the continuum extrapolation. The use of the finite $L$ values of $k$ does not appear to reduce the cutoff effects. The continuum RG flow is shown in 


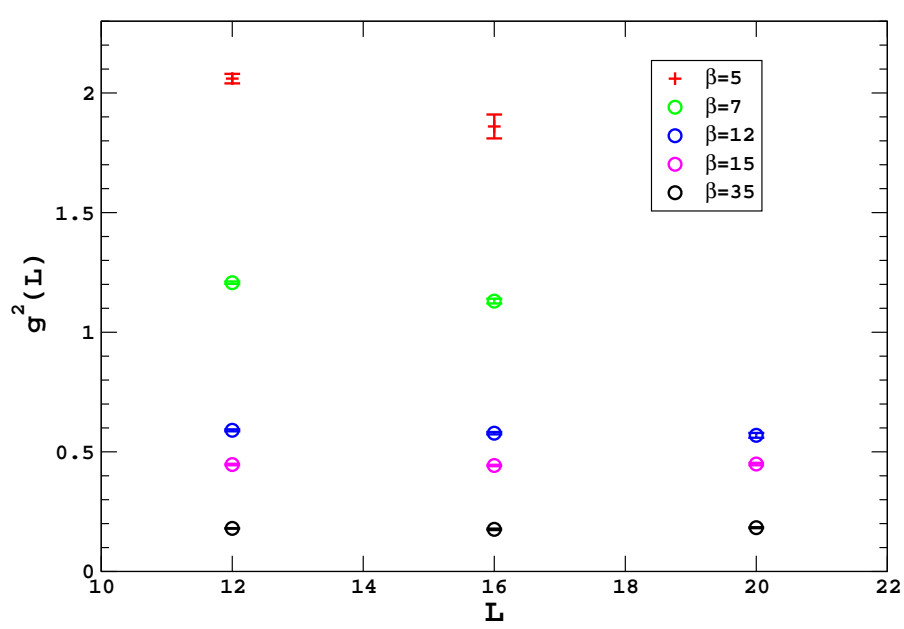

Figure 3: The renormalized coupling $g^{2}(L, \beta)$ at fixed bare coupling for $N_{f}=16$ fundamental flavors. For $g^{2}(L, \beta)>0.5$ the renormalized coupling decreases with increasing $L$, for $g^{2}(L, \beta)<0.5$ the renormalized coupling is independent of $L$ within the errors. This is consistent with the existence of an infrared fixed point.

Fig. 2 (right). At weak coupling, we use analytic/numeric lattice perturbation theory to calculate the Wilson loop ratios in finite volumes [37,38]. The Wilson loops are calculated to 1-loop in the bare coupling in finite volume. The series is reexpanded in the boosted coupling constant at the relevant scale of the Creutz ratio. Step-scaling of the finite volume ratios can be used in exactly the same way as for the simulations results, to determine the RG flow in the continuum. The analytic RG flow starts at the reference point $g^{2}\left(L_{\mathrm{phys}, 0}\right)=0.825$. At weak coupling there is complete agreement with 2-loop perturbation theory. We connect lattice perturbation theory to the simulation results by matching the flows at $g^{2}\left(L_{\text {phys }}\right)=1.44$, where the simulation RG flow begins. There is continued agreement with 2-loop perturbation theory at even stronger coupling, only at the strongest coupling do we see deviation from the perturbative flow.

\section{Fundamental fermions}

We next study $S U(3)$ gauge theory with fermions in the fundamental representation. For $N_{f}=16$ flavors, 2-loop perturbation theory predicts the theory is conformal with an infrared fixed point $g^{* 2} \approx 0.5$. Because of the computational expense of step-scaling with dynamical fermions, in this pilot study we have not yet extrapolated to the continuum limit. The running coupling therefore is still contaminated with finite cutoff effects. If the linear lattice size $L$ is large enough, the trend from the volume dependence of $g^{2}(L, \beta)$ should indicate the location of the fixed point. For $g^{2}(L, \beta)>g^{* 2}$ we expect decrease in the running coupling as $L$ grows, although the cutoff of the flow cannot be removed above the fixed point. Below the fixed point with $g^{2}(L, \beta)<g^{* 2}$ we expect the running coupling to grow as $L$ increases and the continuum limit of the flow could be determined. The first results are shown in Fig. 3. We use stout-smeared [39] staggered fermions $[40,41]$ and the RHMC algorithm, simulating at quark mass $m_{q}=0.01$, with some runs at $m_{q}=$ 
0.001 to test that the mass dependence is negligible. For the Wilson loop ratios, we smear the gauge fields and measure the fat-link Wilson operator. Our experience in the pure gauge theory test is that cutoff dependence is not reduced using the finite $L$ value of $k$, hence we use the infinite volume $k$ value to convert the Wilson loop ratios to a renormalized coupling. The results are consistent with the above picture. For $g^{2}(L, \beta)>0.5$, the cutoff dependent renormalized coupling decreases with $L$. For $g^{2}(L, \beta)<0.5$, the renormalized coupling is $L$-independent within errors. The theory appears conformal but precise determination of the conformal fixed point requires further studies.

\section{Acknowledgments}

We wish to thank Urs Heller for the use of his code to calculate Wilson loops in lattice perturbation theory, and Paul Mackenzie for related discussions. We are grateful to Sandor Katz and Kalman Szabo for helping us in using the Wuppertal RHMC code. In some calculations we use the publicly available MILC code, and the simulations were performed on computing clusters at Fermilab, under the auspices of USQCD and SciDAC, and on the Wuppertal GPU cluster. This research is supported by the NSF under grant 0704171, by the DOE under grants DOE-FG03-97ER40546, DOE-FG-02-97ER25308, by the DFG under grant FO 502/1 and by SFB-TR/55.

\section{References}

[1] S. Weinberg, Phys. Rev. D 19, 1277 (1979).

[2] L. Susskind, Phys. Rev. D 20, 2619 (1979).

[3] E. Farhi and L. Susskind, Phys. Rept. 74, 277 (1981).

[4] F. Sannino and K. Tuominen, Phys. Rev. D 71, 051901 (2005)

[5] D. D. Dietrich, F. Sannino and K. Tuominen, Phys. Rev. D 72, 055001 (2005)

[6] D. D. Dietrich and F. Sannino, Phys. Rev. D 75, 085018 (2007).

[7] T. A. Ryttov and F. Sannino, Phys. Rev. D 76, 105004 (2007).

[8] D. K. Hong, S. D. H. Hsu and F. Sannino, Phys. Lett. B 597, 89 (2004).

[9] H. Georgi, Phys. Rev. Lett. 98, 221601 (2007).

[10] M. A. Luty and T. Okui, JHEP 0609, 070 (2006).

[11] W. E. Caswell, Phys. Rev. Lett. 33, 244 (1974).

[12] T. Banks and A. Zaks, Nucl. Phys. B 196, 189 (1982).

[13] T. Appelquist, K. D. Lane and U. Mahanta, Phys. Rev. Lett. 61, 1553 (1988).

[14] A. G. Cohen and H. Georgi, Nucl. Phys. B 314, 7 (1989).

[15] T. Appelquist, J. Terning and L. C. R. Wijewardhana, Phys. Rev. Lett. 77, 1214 (1996).

[16] B. Holdom, Phys. Rev. D 24, 1441 (1981).

[17] K. Yamawaki, M. Bando and K. i. Matumoto, Phys. Rev. Lett. 56, 1335 (1986).

[18] T. W. Appelquist, D. Karabali and L. C. R. Wijewardhana, Phys. Rev. Lett. 57, 957 (1986). 
[19] V. A. Miransky and K. Yamawaki, Phys. Rev. D 55, 5051 (1997).

[20] E. Eichten and K. D. Lane, Phys. Lett. B 90, 125 (1980).

[21] T. Appelquist, M. Piai and R. Shrock, Phys. Rev. D 69, 015002 (2004)

[22] T. Appelquist, G. T. Fleming and E. T. Neil, Phys. Rev. Lett. 100, 171607 (2008)

[23] S. Catterall, J. Giedt, F. Sannino and J. Schneible, JHEP 0811, 009 (2008)

[24] Z. Fodor, K. Holland, J. Kuti, D. Nogradi and C. Schroeder, Phys. Lett. B 681, 353 (2009)

[25] A. J. Hietanen, J. Rantaharju, K. Rummukainen and K. Tuominen, JHEP 0905, 025 (2009)

[26] A. Deuzeman, M. P. Lombardo and E. Pallante, arXiv:0904.4662 [hep-ph].

[27] L. Del Debbio, B. Lucini, A. Patella, C. Pica and A. Rago, arXiv:0907.3896 [hep-lat].

[28] Y. Shamir, B. Svetitsky and T. DeGrand, Phys. Rev. D 78, 031502 (2008)

[29] A. Hasenfratz, Phys. Rev. D 80, 034505 (2009)

[30] X. Y. Jin and R. D. Mawhinney, PoS LATTICE2008, 059 (2008)

[31] D. K. Sinclair and J. B. Kogut, arXiv:0909.2019 [hep-lat].

[32] E. Bilgici et al., arXiv:0902.3768 [hep-lat].

[33] M. Campostrini, P. Rossi and E. Vicari, Phys. Lett. B 349, 499 (1995)

[34] M. Creutz, Phys. Rev. D 21, 2308 (1980).

[35] A. Bazavov et al., arXiv:0903.3598 [hep-lat].

[36] C. W. Bernard et al., Phys. Rev. D 62, 034503 (2000)

[37] U. M. Heller and F. Karsch, Nucl. Phys. B 251, 254 (1985).

[38] G. P. Lepage and P. B. Mackenzie, Phys. Rev. D 48, 2250 (1993)

[39] C. Morningstar and M. J. Peardon, Phys. Rev. D 69, 054501 (2004)

[40] Y. Aoki, Z. Fodor, S. D. Katz and K. K. Szabo, JHEP 0601, 089 (2006)

[41] Y. Aoki, Z. Fodor, S. D. Katz and K. K. Szabo, Phys. Lett. B 643, 46 (2006) 